Emmanuel Stip Roger Godbout

\section{ADRESSE}

E. Stip : docteur en médecine, maître en sciences, professeur adjoint de clinique. R. Godbout : docteur ès sciences, professeur agrégé. Département de psychiatrie, université de Montréal centre de recherche hôpital du Sacré-Cour de Montréal, 5400, boulevard Gouin ouest, Montréal, Québec, H4J 1C5, Canada.

\title{
Cortex préfrontal, monoamines et neuropsychologie de la schizophrénie
}

Le cortex préfrontal joue un rôle de premier plan dans l'organisation des informations sensorielles à l'origine des comportements et des émotions et dans les processus cognitifs fondés sur l'attention sélective. C'est le lieu de convergence des principales voies ascendantes des systèmes monoaminergiques, dont les neurotransmetteurs sont la dopamine, la sérotonine et la noradrénaline. Cet agencement permet la régulation de l'activité d'un neurone par plusieurs neurotransmetteurs; chacun d'entre eux détermine, à sa manière, si les neurones du cortex préfrontal répondront aux messages afférents. A l'état éveillé normal, les messages essentiels sont ainsi distingués des événements accessoires et cette capacité de discrimination est défectueuse dans les désordres mentaux telle la schizophrénie. Parmi les neuroleptiques, les antipsychotiques atypiques qui agissent spécifiquement sur les voies dopaminergiques et sérotoninergiques semblent capables de restaurer la capacité de traitement de l'information.

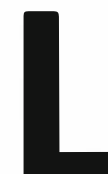
e cortex préfrontal médian (CPF) est une zone corticale associative impliquée dans l'organisation temporelle du comportement et dans la régulation des émotions [1, 2]. Son rôle spécifique est de relever, analyser et stocker momentanément l'information sensorielle, spatiale et temporelle, puis d'intégrer cette information afin de produire des émotions et des comportements qui sont appropriés aux contingences de l'environnement. Il semblerait que le CPF s'aquitte de cette tâche en jouant un rôle dans l'attention sélective, c'est-à-dire en contrôlant l'accès des messages sensoriels aux structures cérébrales supérieures, incluant le CPF lui-même. Le CPF donne ainsi à l'individu la capacité de mettre en relation plusieurs éléments d'information tout en filtrant les informations non pertinentes.

En neuropsychologie, on mesure la capacité du CPF de résister aux interférences en faisant intervenir des éléments distracteurs de façon pro- ou rétroactive au cours de différents tests. On peut ainsi mesurer la capa- 


\section{RÉFÉRENCES}

1. Fuster JM. The prefrontal contex. New York: Raven Press, 1986.

2. Kolb B. Functions of the frontal cortex of the rat: a comparative review. Brain Res Ret $1984: 8: 65-98$.

3. Beckstead RM. An antoradiographic examination of cortico-cortical and subcortical projections of the mediodorsal-projection (prefiontal) cortex in the rat. I Comp) Neurol $1979: 184: 43-62$.

4. Ferino F, Thicery $\wedge$ M, Saffroy M, Glowinski J. Interhemispheric and subcortical collaterals of medial prefiontal cortical neurons in the rat. Brain Res 1987;417:257-65.

5. Auder MA, Doncer (;. Oleskevich S, Descarries L. Quantified regional and laminar distribution of the noradrenaline imnervation in the anterior half of the adult rat cerebral cortex. I Comp Newol 1988; 274: $307-18$.

6. Moore RY, Card JP. Noradrenaline-containing neurons svstems. In: Björklund A, Hökfelt T, eds. Handbook of chemiral neuroanatomy, vol. 2. Amsterdam: Elserier, $1984: 123-56$.

7. Björklund A, Lindlall O. Dopaminecontaining svstems in the CNS. In: Björklund A, Hökfelt T, eds. Handbook of chemical neuroanatomy, vol. 2. Amsterdam: Elscvier. $1984: 55-122$.

8. Descarries L, Lemay B, Doucet (; Berger B. Regional and laminar density of the dopamine inneration in adult rat cerebral cortex. Neuroscience 1987: 21: 807-24.

9. Audet MA, Descarries L, Doucet (i. Quantified regional and laminar distribution of the serotonin inneration in the anterior half of adult rat cerebral cortex. I Chem Neuroanat 1989: 2: 29-44.

10. Steinbusch HWM. Serotonin-immunoreactive neurons and their projections in the CNS. In : Björklund A, Hökfelt T, Kuhar MJ, eds. Handbook of chemiral neuroamatomy. cité d'un individu de maintenir un objectif en vérifiant s'il peut respecter un ensemble de contraintes imposées avant l'exécution d'une tâche. L.es fonctions du C.PF peuvent aussi être étudiées chez l'animal. (On observe par exemple quim singe auquel on a fait une ablation du C.PF éprouvera de grandes difficultés pour apprendre une tâche slimulus. réponse dont les indices sensoriels renforçateurs et non renforçateurs sont présentés l'un après l'autre, à intervalle variable. C"est le cas des tâches dites de type " $g(0) / m(r-g())$, où l'un des indices doit être suivi de la bonne réponse alors que l'autre ne doit être suivi d'aucune réponse, quelle quielle soit. Dans de tels cas, l'animal lésé persiste à produire des erreurs de commission: il domnera par exemple une réponse lors de l’indice " $\left.n(1)-g_{0}\right) "$ il sera distrait et réagira sans discrimination à toute stimulation non spécifique venant de l'envirommement et non reliéc à la tâche (hỵperréactivité à l'environnement et à la nouveauté). De plus, lorsque l'animal aura finalement réussi à apprendre une telle tâche, il montrera une grande difficulté à se détacher du comportement appris et ne saura pas apprendre la tâche inverse (où les indices " $\left.g_{0}\right)$ " et " $n(1)-g()$ " sont intervertis). I.es mêmes phénomènes de distraction et de persévération* sont observés chez les rats dont le (.PF a été lésé [2].

I.es manifestations comportementales du (.PF ne se limitent pas au paradigme des tests d'apprentissage. Le CPF joue également un rôle important dans la performance psichomotrice de même que dans la régulation des états émotionnels. Dans ces deux cas, le processus sera le même que ce qui a été décrit ci-dessus: après une analyse des contingences de l'envirominement, le C.PF permettra la manifestation du comportement le plus approprié au contexte, que ce soit la séquence de mourements nécessaires pour atteindre un but préalablement fixé (la recherche de nourriture, par exemple), ou la réaction émotionnelle adaptéc au contexte (par exemple l’agressivité). I.es prochains paragraphes décrivent brièvement les sub-

\footnotetext{
* Terme général indiquant le maintien d une attitude, la repritition diun geste ou de mots.
}

strats neuroanatomiques à la base de la communication nerveuse dans le (.PF, notamment son innervation par les neurotransmetteurs monoaminergiques.

\section{Transmission synaptique dans le cortex préfrontal}

L'organisation neuroanatomique des efférences et des afférences du C.PF est à la base du rôle intégrateur que l'on attribue à cette région corticale. Grâce à ses axones fortement branchés, le C.PF entretient en effet de multiples commexions avec d'autres structures corticales et sous-corticales, ce qui en fait une rone de convergence dotée d'un très grand potentiel d'intégration physiologique [3, 4]. De prime abord, on peut définir anatomiquement les limites du C.PF, chez tous les mammifères, comme étant la zone de projection néocorticale du novau dorsomédian du thalamus. Par ailleurs, le (.PF s'avère être aussi une région du cortex où convergent les trois systèmes monoaminergiques ascendants, noradrénergique, dopaminergique et sérotoninergique (5-HT). I.c type d’agencement que présentent ces trois sustemes d'innervation dans le (.PF est compatible avec le concept d'hétérorégulation de l'activité d'un même neurone du (.PF par plusieurs ncurotransmetteurs à la fois.

I. immervation noradrénergique du (.PF est issue du locus coeruleus. Un premier groupe de fibres traverse de façon diffuse toutes les couches laminaires du C.PF, avec une densité plus élevée dans la couche I $[5,6]$. Il existe un second groupe de fibres dotées d'une orientation tangentielle leur permettant de parcourir sur de longues distances une même couche laminaire; ces fibres parallèles se retrouvent surtout dans les couches I et I'I du (.PF. Cette organisation permet à la noradrénaline d'assurer la transmission nerveuse dans de larges régions corticales et d'en affecter l'excitabilité neuronale. I.e territoire du (.PF innervé par le système dopaminergique est, au contraire, restreint. Issue principalement de l'aire tegmentaire ventrale, cette innervation se limite aux couches corticales profondes (V et VI), dans une région entourant le pôle antérieur du corps calleux et qui s'étend médialement jusqu'au cortex cingulaire $[7,8]$. En- 


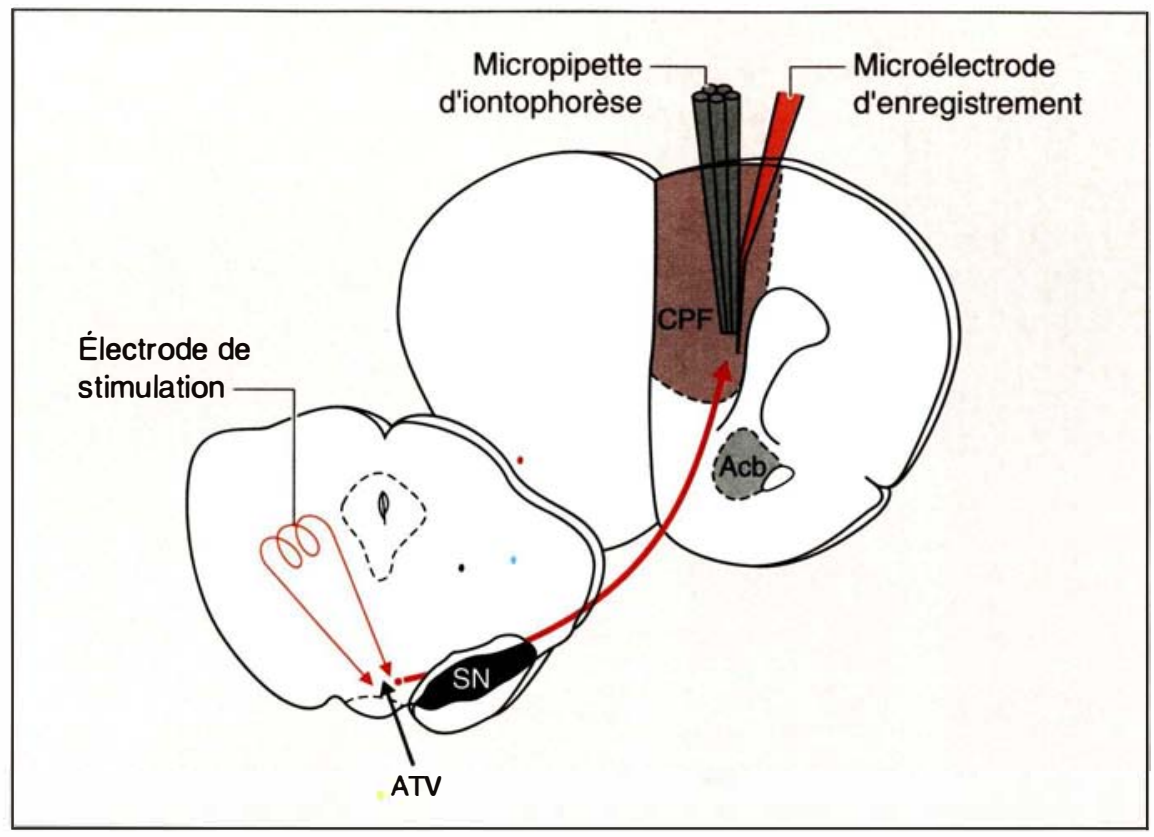

Figure 1. Dispositif expérimental d'étude électrophysiologique du cortex préfrontal. Représentation schématisée de coupes coronales au niveau de l'aire tegmentaire ventrale (ATV) et du cortex préfrontal médian (CPF) illustrant la disposition des électrodes d'enregistrement, de micro-iontophorèse et de stimulation. Le rat anesthésié est placé dans un appareil stéréotaxique. La microélectrode d'enregistrement, remplie de solution saline, a une pointe de diamètre 5-8 $\mu \mathrm{m}$, permettant de n'étudier qu'un seul neurone. On peut lui accoler une micropipette à plusieurs canaux, permettant l'injection dans son voisinage immédiat des substances pharmacologiques testées. La microélectrode de stimulation (pointe de diamètre $\simeq 200 \mu \mathrm{m}$ ), placée au niveau de l'aire tegmentaire ventrale, provoque la libération de neurotransmetteurs endogènes auprès du neurone enregistré. Acb : noyau accumbens; SN : substance noire.

fin, l'innervation par le système sérotoninergique du CPF est issue des noyaux mésencéphaliques du raphé, le raphé dorsal et le raphé médian [9, 10]. Les fibres sont sinueuses, fortement arborisées et occupent toutes les couches corticales avec une densité plus grande que celle des fibres noradrénergiques. Les fibres 5-HT semblent se distribuer sur le plan laminaire en fonction de leur orientation, de sorte que l'on retrouve une densité particulièrement forte de fibres tangentielles dans la couche I et la couche VI. Cette organisation permet aux fibres 5-HT de se juxtaposer aux terminaisons à noradrénaline et à dopamine $[5,10]$.

Ces caractéristiques montrent que le CPF est un lieu de convergence privilégié pour la communication des mes- la neurotransmission monoaminergique. En appliquant à l'un de ces canaux des courants de l'ordre du nanoampère et de même polarité que la solution ionisée, il est possible par la technique de micro-iontophorèse d'injecter dans le voisinage immédiat du neurone enregistré des quantités infimes de substances pharmacologiques et d'en mesurer les eff ets sur l'activité électrique (fréquence de décharge) de ce même neurone. Enfin, par une électrode de stimulation intracérébrale (diamètre de la pointe: 200 microns), on peut aussi provoquer la libération de neurotransmetteurs endogènes dans le voisinage immédiat du neurone enregistré en stimulant électriquement (ondes carrées de 0,5 milliseconde à $100-500 \mathrm{mi}-$ cro-ampères) les noyaux monoaminergiques qui l'innervent. Dans ces conditions, on peut évaluer l'effet de la dopamine, de la 5-HT et de la noradrénaline sur deux types d'activité neuronale dans le CPF, l'activité spontanée et l'activité évoquée [11].

La stimulation électrique de l'aire tegmentaire ventrale (associée à la dopamine), des noyaux mésencéphaliques du raphé (associés à la 5-HT) et du locus coeruleus (associé à la noradrénaline) est capable d'inhiber l'activité spontanée des neurones du CPF et le fait pour chacune de façon spécifique. La stimulation de l'aire tegmentaire ventrale $[12,13]$ ou des noyaux du raphé [14] à la fréquence de $1 \mathrm{~Hz}$ induit, dans $50 \%$ à $80 \%$ des neurones du CPF enregistrés, un silence total mais transitoire dans la fréquence de décharge, soit pendant 75 à 150 millisecondes (figure 2). L'effet inhibiteur de la stimulation de l'aire tegmentaire ventrale disparaît sélectivement après lésion neurotoxique spécifique de la voie dopaminergique ascendante ou lorsque l'animal a reçu préalablement une injection d'un antagoniste des récepteurs dopaminergiques de la famille $\mathrm{D}_{2}$ (et notamment des neuroleptiques de type sulpiride et fluphénazine). Par ailleurs, l'application par micro-iontophorèse de ces mêmes antagonistes bloque à la fois l'effet inhibiteur de la stimulation de l'aire tegmentaire ventrale et l'inhibition induite par l'application micro-iontophorétique de dopamine; dans tous les cas, les ligands de la famille de récepteurs de type $\mathrm{D}_{1}$ (SCH-23390) 


\section{RÉFÉRENCES}

11. Thierry AM, Godbout R, Mantz J, Glowinski $J$. Influence of the ascending monoaminergic svstems on the activity of the rat prefrontal cortex. Prog Brain Res 1990); 85 : $357-65$

12. Ferron A, Thierry AM, Le Douarin C, Glowinski J. Inhibitory influence of the mesocortical dopaminergic system on spontaneous activity or excitatory response induced from the thalamic mediodorsal nucleus in the rat medial prefrontal cortex. Brain Res $1984 ; 302: 257-65$.

13. Godbout R, Mantz J, Pirot S, Glowinski $J$, Thierry AM. Inhibitory influence of the mesocortical dopaminergic neurons on their target cells: electrophysiological and pharmacological characterization. J Pharmacol Exp Ther 1991 ; 258: 728-38.

14. Mant7. J, Godbout R, Tassin JP, Glowinski J. Thierny AM. Inhibition of spontaneous and evoked activity in the rat medial prefrontal cortex by mesencephalic raphe nuclei. Brain Res 1990; 524: 22-30.

15. Godbout R, Manc. J, Glowinski J, Thierry AM. The novel 5 -HT receptor antagonist, RP 62203, selectively blocks serotoninergic but not dopaminergic-induced inhibition in the rat prefrontal cortex. Eur I Pharmacol $1991 ; 204: 97-100$

16. Mantz J, Milla (;, Glowinski J. Thierry AM. Differential effects of ascending neurons containing dopamine and noradrenaline in the control of spontaneous activity and of evoked responses in the rat prefrontal cortex. Neuroscience 1988; $27: 517-26$.

17. Mantz.J, Thierry AM, Glowinski J. Effects of noxious tail pinch on the discharge rate of mesocortical and mesolimbic dopamine neurons: selective activation of the mesocortical svstem. Bruin RPs $1989 ; 476: 377-81$.

18. Condès-Lara M, Omaña Zapata I, Léon-Olea M. Sancher-Alvarez M. Dorsal raphe and nociceptive stimulations evoke convergent responses on the thalamic centralis lateralis and medial prefrontal cortex neurons. Brain Res 1989; 499 : 145-52.

19. Dworkin RH. Pain insensitivity in schizophrenia: a neglected phenomenon and some implications. Schizophr Bull 1994: 20: $235-48$.

20. Petersen SE, Fox PT, Snyder AZ, Raichle ME Activation of extrastriate and frontal cortical areas by visual words and word-like

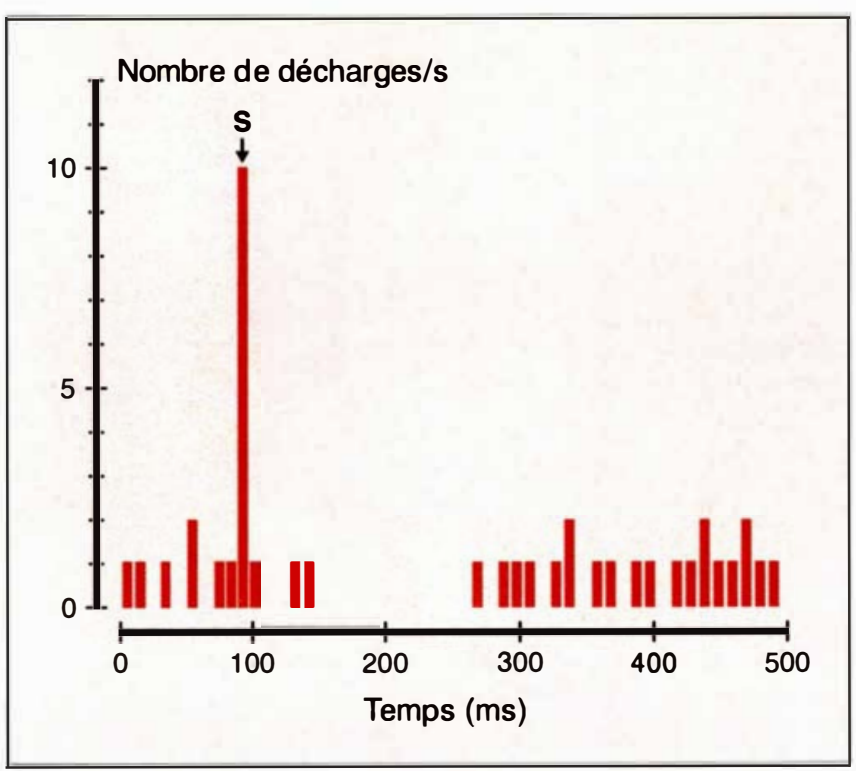

Figure 2. Décharges neuronales accumulées lors de 20 stimulations de l'aire tegmentaire ventrale à la fréquence de $1 \mathrm{~Hz}$ (délivrées au moment " $S$ »). Histogramme de fréquences cumulées (données fictives). Le bref silence neuronal qui apparait approximativement entre 150 et 280 ms après la stimulation de l'aire tegmentaire ventrale est attribué à l'effet inhibiteur de la libération de dopamine endogène dans la fente synaptique. Pendant ce silence, la cellule du CPF ne réagit plus aux messages excitateurs qui lui parviennent du thalamus dorsomédian.

sont sans effet significatif. Dans le cas de l'effet inhibiteur de la stimulation des noyaux du raphé, celui-ci disparaît après une lésion neurotoxique sélective de la voie 5 -HT par la 5,7 -dihydroxytryptamine ou encore après une injection d'un antagoniste des récepteurs sérotoninergiques de la famille $5-\mathrm{HT}_{2 \mathrm{~A}}$ (kétansérine, ritansérine, RP-62203). En outre, nous avons pu montrer qu'un agoniste des récepteurs 5-HT tel l'hallucinogène LSD peut potentialiser l'inhibition des neurones du CPF induite par la stimulation de l'aire tegmentaire ventrale (associée à la dopamine); nous avons pu également montrer que cet effet peut être inversé par le RP-62203, un nouvel antagoniste des récepteurs $5-\mathrm{HT}_{2}$ [15]. Le LSD étant un agoniste des récepteurs 5-HT, on peut suggérer, d'une part, l'existence dans le C.PF d'une potentialisation de l'effet inhibiteur de la dopamine par la 5-HT et, d'autre part, la capacité pour les antagonistes $5-\mathrm{HT}_{2}$ de bloquer une facilitation anormale de l'inhibition du CPF par la dopamine. Dans le cas du locus coeruleus (associé à la noradrénaline), il faut appliquer un train de pulsions de fréquence $20 \mathrm{~Hz}$ pendant 10 secondes consécutives avant d'inhiber l'activité de $60 \%$ des neurones du CPF [16] : la stimulation induit une inhibition soutenue de la fréquence de décharge, pendant 40 à 50 secondes (figure 3). La lésion neurotoxique sélective des voies noradrénergiques par la neurotoxine 6-hydroxydopamine ou la déplétion des catécholamines par l'administration d' $\alpha$-méthyl-para-tyrosine s'accompagne de la disparition presque totale de l'effet inhibiteur de la stimulation du locus coeruleus. Par ailleurs, l'effet inhibiteur de la noradrénaline sur l'activité du CPF disparaît totalement lors de la co-application micro-iontophorétique d'un antagoniste des récepteurs $\beta$-adrénergiques (propranolol) alors que les antagonistes des récepteurs $\alpha_{1}$ (prazosin) et $\alpha_{2}$ (yohimbine) sont sans effet [13].

Ces travaux montrent donc que la li- 


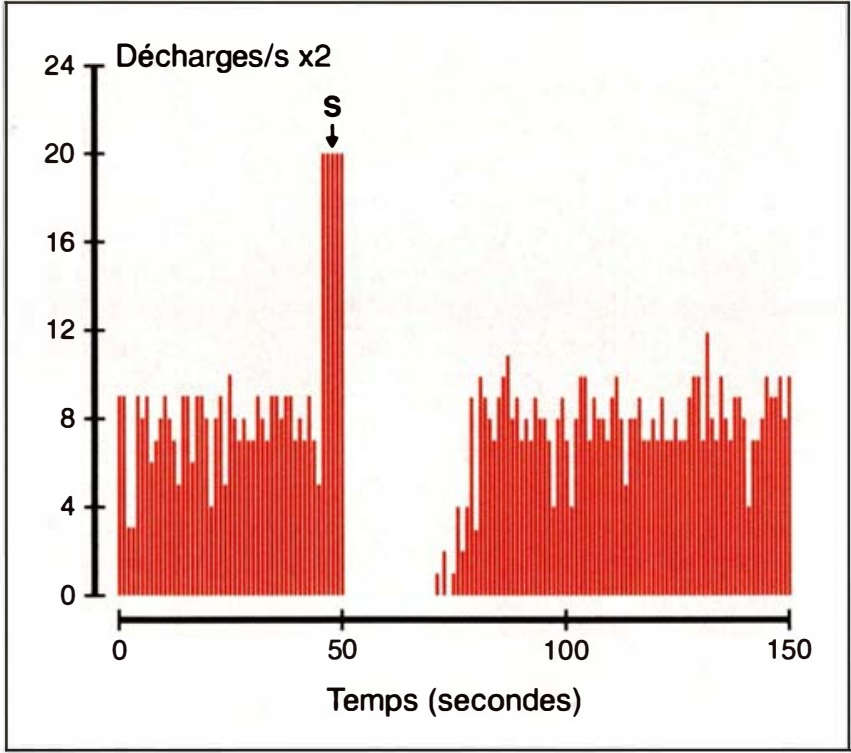

Figure 3. Effet d'une stimulation du locus cœruleus à la fréquence de $20 \mathrm{~Hz}$ pendant 10 s (délivrée au moment S) sur l'activité d'une cellule du CPF. Histogramme de fréquences continu (données fictives). Le silence neuronal de plusieurs dizaines de secondes qui apparaît au milieu du tracé est attribué à l'effet inhibiteur de la libération de noradrénaline endogène dans la fente synaptique. Contrairement à l'effet de la stimulation du raphé et de l'aire tegmentaire ventrale, la cellule du CPF continue de pouvoir réagir aux messages excitateurs qui lui parviennent pendant la période d'inhibition.

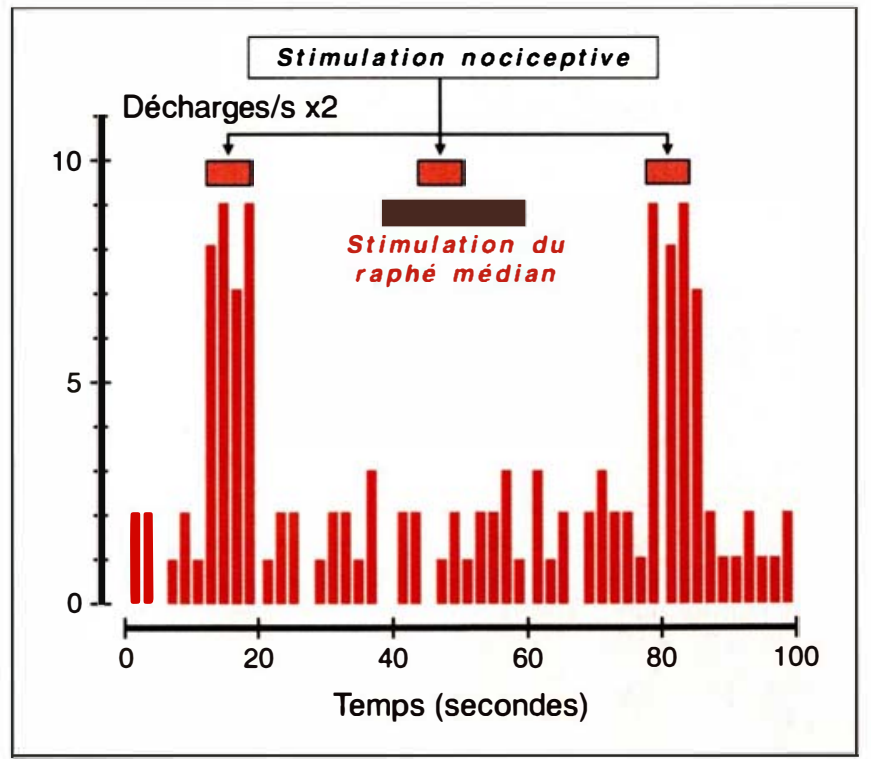

Figure 4. Activité d'une cellule du CPF évoquée par une stimulation nociceptive (pincement de la queue) chez le rat anesthésié. Histogramme de fréquences continu (données fictives). Cette réponse évoquée apparait à la $14^{e}$ et à la $80^{\circ}$ seconde mais ne peut apparaitre entre la $40^{\circ}$ et la $60^{\circ}$ seconde, au moment où l'on stimule le raphé médian à la fréquence de $10 \mathrm{~Hz}$ (rectangle bistre). bération de dopamine et de 5-HT sur les neurones du CPF induit chez. ceux-ci une inhibition phasique (transitoire) de l'activité neuronale alors que la libération de noradrénaline serait plutôt associée à une inhibition tonique (prolongée) de cette activité. Les systèmes monoaminergiques ascendants ont également une influence permissive sur l'apparition de réponses excitatrices évoquées dans le CPF. De telles réponses peuvent être évoquées chez le rat anesthésié. La stimulation électrique du noyau thalamique dorsomédian à la fréquence de $5-10 \mathrm{~Hz}$ fait apparaître, dans $80 \%$ des cas, une réponse neuronale simple en moins de 20 millisecondes. Une stimulation périphérique douloureuse, tels le pincement de la queue ou l'immersion de la queue dans l'eau chaude pendant une dizaine de secondes, fait apparaître, pour au moins la durée de la stimulation et parfois même plus, une importante augmentation de la fréquence de décharge dans $25 \%$ des neurones du C.PF. Ce type de protocole s'avère particulièrement intéressant car il permet de caractériser la façon dont le C.PF traite l'information afférente dans différentes conditions expérimentales. Nous avons pu montrer que la stimulation de l'aire tegmentaire ventrale et des noyaux du raphé est capable de bloquer l'apparition des réponses excitatrices évoquées dans le CPF alors que l'activité spontanée de base peut demeurer intacte (figure 4) $[14,16]$. En revanche, la stimulation du locus coeruleus ne modifie pas la probabilité d'apparition des réponses évoquées alors qu'elle inhibe l'activité spontanée de base pour une période prolongée (voir figure 3) [16]. Dans aucun de ces cas, le type de récepteur impliqué n'a été identifié avec certitude. Ces résultats suggèrent deux niveaux d'interprétation. A un premier niveau, ils montrent, d'une part, que lorsque la voie noradrénergique ascendante est activée au moment même où le CPF est excité par une information afférente, la valeur du message excitateur est augmentée du fait que le locus coeruleus inhibe de façon tonique l'activité de base du neurone sans modifier la valeur du message afférent. En d'autres termes, la noradrénaline augmenterait dans le CPF la valeur du rapport signal/bruit. D'autre part, l'activa- 


\section{RÉFÉRENCES}

21. Weinberger DR, Berman KF, Zec RF. Physiologic dysfunction of dorsolateral prefrontal cortex in schizophrenia. Arch Gen Psychiatr 1986; 43: 114-35.

22. Berman KF, Ostrem JL, Van Horn JD, Mattay VS, Esposito G, Weinberger DR. A comparison between normal monozygotic and dizvgotic twins studied during cognition with positron emission tomography. Soc Neurositi Abstr 1993; 19: 792

23. Kraepelin E. Dementia Praecox and Paraphrenia (traduit par Barclay RM). New York : Krieger, 1919/1971.

24 Liddle PF. The symptoms of chronic schizophrenia: a reexamination of the positive-negative dichotomy. $\mathrm{Br}$ J Psychiatr 1987; $151: 147-51$

25. Goldman-Rakic PS, Working memory dysfunction in schizophrenia. I Neuropsychiatry Clin Neurosei 1994:6: 348-57.

26. Cohen RM, Sample WE, Gross M, Nordhal TE, Holcomb HH, Dowling MS, Pickar D. The effect of neuroleptics on dysfunction substrate of sustained attention in schizophrenia. I.ife Sci 1988; 43: 1141-50.

27. Crider A, Solomon PR, McMahon MA. Disruption of selective attention in the rat following d-amphetamine administration: relationship to schizophrenic attention disorder. Biol Psychiatr 1982; 17 : 357-61.

28. Arguin M, Joanette Y, Cavanagh P. Visual search for feature and conjunction targets with an attention deficit. J Cogn Neurosi $1993 ; 5: 4$

29. Meltzer HY, Matsubara S, Lee JC. Classification of typical and atypical antipsychotic drugs on the basis of dopamine D-1, D-2

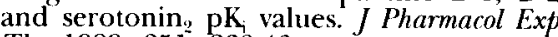
Ther 1989; 251 : 238-46.

30. Tassin JP. NE/DA interactions in prefrontal cortex and their possible roles as neuromodulators in schizophrenia. / Neurol Transm 1992; 36 (suppl) : 135-62.

31. Van Kammen DP, Kelley M. Dopamine and norepineprine activity in schizophrenia. An integrative perspective. Schizophr Res tion des voies dopaminergiques et 5-HT ascendantes peut diminuer la valeur absolue d'un message afférent sans affecter l'activité neuronale de fond. Il en découle que l'activation concomitante des trois systèmes monoaminergiques ascendants peut contribuer à établir un ordre de priorité dans les informations à traiter par le CPF. A un second niveau, plus hypothétique pour l'instant, ces résultats pourraient aider à mieux comprendre les substrats qui sous-tendent l'interprétation affective des messages nociceptifs. On peut imaginer en effet que le recrutement des systèmes méso-corticaux dopaminergiques [17] et 5-HT [18] qui accompagne une stimulation nociceptive viserait à moduler l'interprétation du message douloureux par le CPF. L'effet excitateur de la morphine et autres opiacés sur l'activité neuronale spontanée de l'aire tegmentaire ventrale et du raphé n'est peut-être pas étranger à leur propriété analgésique. Dans les troubles psychiatriques où l'on soupçonne une anomalie dans la neurotransmission monoaminergique ascendante et une hypofrontalité, comme dans la schizophrénie, on devrait donc s'attendre à un syndrome d'indifférence aux messages douloureux car les informations afférentes signifiantes et les stimuli non pertinents ne sont plus discriminés. Cette observation clinique familière a récemment été discutée dans la littérature [19].

Bien sûr, plusieurs autres neurotransmetteurs/neuromodulateurs participent au contrôle du transit de l'information au niveau du CPF, notamment l'acétylcholine, les acides aminés excitateurs, le GABA et les neuropeptides. En outre, d'autres sites cérébraux voient également leur activité modulée par les monoamines. L’intérêt plus particulier que revêt la compréhension de la modulation de l'activité neuronale du CPF par les monoamines réside dans le fait que ces systèmes de neurotransmission sont la cible privilégiée des substances psychotropes telles que les neuroleptiques, les antidépresseurs, les psychostimulants et les substances hallucinogènes.

Dans les prochains paragraphes, nous allons donc revoir certaines études qui relient la symptomatologie d'un trouble de la pensée, la schi- zophrénie, au CPF de même qu'à son innervation par les voies monoaminergiques. Ce lien sera illustré par la présentation des résultats d'une étude pilote concernant l'action de neuroleptiques atypiques ayant des propriétés à la fois antidopaminergiques et antisérotoninergiques, la clozapine et le rispéridone. Compte tenu des effets de la dopamine et de la 5-HT sur le traitement de l'information par le CPF tels que décrits plus haut, ces molécules devraient être capables de restaurer l'attention sélective chez des patients schizophrènes, c'est-à-dire d'améliorer leur capacité de détecter un signal-cible parmi des éléments distracteurs.

\section{Le CPF et la schizophrénie}

Confortées par des preuves expérimentales, de nombreuses hypothèses lient la schizophrénie à une anomalie du C.PF. Par exemple, une des dysfonctions cognitives bien connue de la schizophrénie est le syndrome dit "frontal" de cette maladie, principalement caractérisé par de la persévération. Ce trouble caractéristique est souvent mis en évidence par des épreuves comme le Wisconsin card sorting test (WCST), lequel requiert des habiletés d'alternance conceptuelle. On sait que les capacités d'abstraction ou de conceptualisation sont grandement perturbées chez les schizophrènes, ce qui pourrait contribuer à expliquer leurs déficits dans des tâches comme le WCST. Or, le traitement de l'information sémantique lors de la présentation auditive ou visuelle de mots simples est associé à une activation de l'aire dorsolatérale du CPF [20]. Weinberger et al. [21] ont montré que l'augmentation bilatérale du débit sanguin cérébral, normalement observée pendant le WCST chez le sujet sain, est absente chez le patient schizophrène. La tomographie par émission de positons a également permis de mettre en évidence un trouble du CPF chez les patients schizophrènes lors de tâches cognitives [22].

\section{Les symptômes négatifs}

Dans la schizophrénie, les symptômes tels l'apathie, l'incapacité de s'exprimer par la parole (alogie), le caractè- 
re émoussé de l'affectivité, l'autisme et la pauvreté du contenu du langage sont considérés comme des signes négatifs. Ces symptômes sont similaires à l'aboulie (trouble mental caractérisé par une diminution considérable de la volonté) et au mutisme akinétique vus lors des lésions de l'aire motrice supplémentaire et du cortex cingulaire antérieur, ou des projections dopaminergiques ascendantes vers ces régions. Le mutisme akinétique est un état d'extrême avolition (incapacité d'avoir une volonté) et est parfois diagnostiqué par erreur comme de la catatonie (état d'inertie motrice et psychique).

Chez les sujets normaux, le CPF est impliqué dans la mise en route d'une réponse allant de la simple action motrice à l'élaboration de réponses verbales, ce qui permet de réaliser une activité auto-dirigée. Chez les malades schizophrènes qui ont une atteinte de leur capacité de démarrer une action, l'activité du CPF dorso-latéral est réduite. Si la difficulté réside dans l'organisation de l'action, on observe une sous-activité du CPF orbital et une sur-activité au niveau du cortex cingulaire antérieur droit qui est impliqué dans la suppression des réponses inappropriées. Kraepelin [23] a décrit un dysfonctionnement de la volition (faculté d'avoir une volonté) comme une des caractéristiques de la schizophrénie. Liddle [24] a suggéré que cette atteinte de la volition reflétait différents aspects des processus pathologiques de la schizophrénie. Dans une étude sur les relations entre les symptômes schizophréniques, il a séparé ces symptômes en trois syndromes: (1) la pauvreté psychomotrice (pauvreté du discours, affect plat, diminution des mouvements spontanés); (2) la désorganisation (désordre de la forme de la pensée et affect inapproprié); (3) la distorsion de la réalité (délire et hallucinations).

Deux de ces syndromes apparaissent refléter les désordres de la volition: la pauvreté psychomotrice reflète une difficulté de commencer une action et la désorganisation reflète une difficulté dans la sélection d'une activité appropriée. Toutes deux sont associées à une atteinte des tests neuro-psychologiques relatifs aux fonctions du lobe frontal. Selon la proposition de Liddle, le syndrome de pauvreté psychomotrice est relié à une dysfonction du CPF dorso-latéral gauche, le syndrome de désorganisation est relié à une dusfonction du CPF orbital droit et le syndrome de distorsion de la réalité est relié au lobe temporal médian.

Il est possible que des anomalies de la neurotransmission dopaminergique puissent contribuer aux dysfonctions préfrontales qui existent dans la schizophrénie. Goldman-Rakic [25] suggère que la schizophrénie pourrait être conceptualisée comme une rupture dans le processus par lequel le savoir représentationnel dirige les comportements, fonction qui serait sous la responsabilité du CPF. Les lésions qui détruisent les terminaisons dopaminergiques au niveau du CPF produisent des changements cognitifs et de motivation. Cohen et al. [26] ont rapporté une diminution de l'activité métabolique dans le cortex frontal droit (méthode d'investigation au fluoro-désoxyglucose) chez les patients schizophrènes traités par des neuroleptiques au cours d'une tâche de performance de soutien de l'attention à des stimuli auditifs (continuous performance test). Ces résultats contrastaient avec l'augmentation du métabolisme du glucose au niveau de l'hippocampe et des régions sous-corticales telles que les noyaux gris centraux et le thalamus. Le métabolisme du glucose dans le cortex frontal antérieur droit était corrélé positivement à la performance lors du test. Les chercheurs concluaient que la dopamine devait jouer un rôle important dans le soutien de l'attention et suggéraient que les neuroleptiques pouvaient supprimer une activité non reliée à un but dans le cortex frontal. Ils notaient cependant que le traitement neuroleptique n'augmentait pas le métabolisme régional dans le cortex frontal médian dans une aire qui, chez les sujets témoins débutant la même tâche, montrait une augmentation marquée du métabolisme glucosé. Ils postulaient alors que l'effet du traitement neuroleptique est de renforcer le filtre sensoriel au cortex, améliorant ainsi l'attention. Il existe, en effet, des données fondées sur des études cliniques précisant que l'augmentation de l'activité dopaminergique peut entraîner des déficits de l'attention sélective [27].
Neuroleptiques et attention sélective

Il est possible que le CPF puisse exercer un contrôle sur l'activité des neurones sous-corticaux dopaminergiques. Les antipsychotiques atypiques, telle la clozapine, pourraient posséder des caractéristiques spéciales avec un effet primaire sur les mécanismes corticaux et des influences secondaires sur les mécanismes sérotoninergiques et dopaminergiques sous-corticaux. Ia clozapine est un agent antipsychotique puissant, antagoniste à la fois des récepteurs dopaminergiques $\left(\mathrm{D}_{2}, \mathrm{D}_{3}\right)$ et des récepteurs $5-\mathrm{HT}_{2}$ et $5-\mathrm{HT}_{3}$. Le rispéridone est un nouveau neuroleptique atypique, bloquant à la fois les récepteurs $\mathrm{D}_{22}$ et $5-\mathrm{HT}_{22}$. Ces deux neuroleptiques sont susceptibles d'améliorer les signes négatifs de la schizophrénie. Le but de l'étude suivante était d'examiner les fonctions cognitives chez des patients schizophrènes traités par ces neuroleptiques pendant trois mois, afin de déterminer, en particulier, si ces neuroleptiques ont une influence sur l'attention sélective, c’est-à-dire sur la capacité de discriminer une information pertinente d'une information non pertinente.

I.es neuf malades qui ont participé à l'étude avaient reçu le diagnostic de schizophrénie selon les critères DSM-III-R, et prenaient des neuroleptiques conventionnels. Leur âge variait de 22 à 45 ans (movenne 33 ans). Les sýmptômes de la schizophrénie étaient mesurés par le Brief psychiatric rating scale (BPRS) et le Positive and negative syndrome scale (PANSS) alors que les signes extrapyramidaux ont été quantifiés avec l'échelle Extrapyramidal signs rating scale (ESRS). Les patients étaient examinés avant (J0) et trois mois après (Jl) le début du traitement par les neuroleptiques atypiques (rispéridone, $\mathrm{N}=\overline{5}$, dosage moyen quotidien : $6,20 \mathrm{mg}$; clozapine, $\mathrm{N}=4$, dosage moyen : $325 \mathrm{mg}$ ). A J0, six patients recevaient, en outre, une médication antiparkinsonnienne (procyclidine, dosage moyen de $17 \mathrm{mg}$ pour le groupe avant clozapine et de $25 \mathrm{mg}$ pour le groupe avant rispéridone). A J0, le score total PANSS était de 86,11 (écart type: 17,34) et le BPRS était de 34,66 (écart type: 10,07). L'évaluation neuropsychologique des malades était complète mais nous ne dé- 
taillerons ici que l'une des composantes d'attention, l'attention sélective évaluée par une tâche de recherche visuelle [28].

Les sujets devaient rechercher parmi des stimuli présentés sur l'écran d'un ordinateur une cible spécifique définie par la conjonction d'éléments (couleur et forme). La cible était une croix noire, tandis que les stimuli distracteurs étaient des croix blanches, des $\mathrm{O}$ noirs ou des $\mathrm{O}$ blancs. Pour chacun des 80 essais consécutifs composant la tâche, le sujet devait répondre le plus rapidement possible sur un clavier à deux clés quant à la présence ou non de la cible parmi les stimuli. De façon aléatoire, la cible était présente à l'écran dans la moitié des essais et elle en était absente dans l'autre moitié. Un point de fixation était présenté au centre de l'écran pendant une seconde entre chaque essai. Ce point était ensuite remplacé par les stimuli qui demeuraient à l'écran jusqu'à ce que le sujet réponde. La tâche a été présentée sous quatre conditions définies par le nombre de stimuli présentés en même temps à l'écran lors d'un essai, soit $1,4,7$ ou 10 stimuli. L'expérience était conduite dans une salle calme adaptée. Les temps de réaction et les taux d'erreur des sujets étaient enregistrés pour chaque facteur (présence ou non de la cible et nombre total de stimuli à l'écran). Les résultats du groupe de patients schizophrènes à J0 ont été comparés aux résultats normatifs d'un groupe de sujets sains fournis par l'équipe du Centre de recherche de l'hôpital Côte-des-Neiges à Montréal, responsable de la mise au point des logiciels d'expérimentation. Les résultats du groupe de patients schizophrènes à J0 ont également été comparés aux résultats obtenus à $\mathrm{J} 1$.

Sous clozapine ou rispéridone, tous les patients ont montré une amélioration clinique significative définie par une baisse du score supérieur à $20 \%$ au PANSS et au BPRS. Une analyse de variance à trois facteurs (Groupe/nombre d'items à l'écran/ présence de la cible) fut effectuée sur la médiane du temps de réaction et révéla une interaction statistique significative entre le groupe et la présence de la cible $[F(1,17)=3,23$; $\mathrm{p}=0,09]$. Comme le montre la figu-

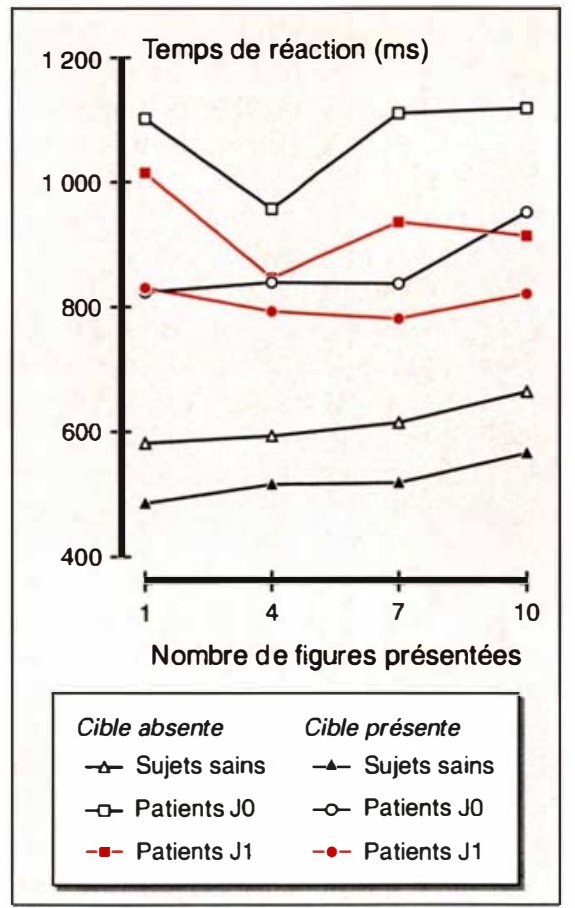

Figure 5. Épreuve d'attention sélective chez des patients présentant une schizophrénie et recevant des neuroleptiques atypiques. L'attention sélective était mesurée par une épreuve de recherche visuelle d'une cible parmi des distracteurs. On a mesuré le temps de réaction et calculé la médiane pour l'ensemble des neufs sujets schizophrènes et des sujets issus d'un groupe témoin composé de sujets sains. Le temps de réaction est toujours plus court quand la cible est présente parmi les autres figures présentées à l'écran. Les schizophrènes ont fait la tâche avant l'introduction (JO) du neuroleptique atypique (clozapine ou rispéridone) et trois mois après (J1). Ils améliorent leur performance d'attention sélective avec le neuroleptique atypique et se rapprochent des sujets sains.

senté un temps de réaction plus lent que les sujets sains. En revanche, sous traitement par un neuroleptique atypique, ces mêmes patients ont amélioré significativement leur temps de réaction et se sont approchés des résultats des sujets sains. Parmi toutes les autres mesures cognitives que nous avons effectuées, nous avons observé que le temps de réaction est la seule composante qui s'améliore en même temps que l'état clinique. L'administration de neuroleptiques atypiques n'altère pas le fonctionnement cognitif et des résultats préliminaires indiquent qu'il est possible de rendre compte de modifications cognitives, ici d'attention sélective, dans une maladie aussi complexe que la schizophrénie pour laquelle la classique hypothèse étiologique dopaminergique n'est plus suffisante.

Selon Meltzer [29], la meilleure garantie qu'un nouveau neuroleptique peut offrir quant à sa capacité d'induire les effets thérapeutiques les plus puissants tout en provoquant des effets secondaires extrapyramidaux les plus restreints est de maximiser le rapport de ses affinités antagonistes respectives pour les récepteurs $5-\mathrm{HT}_{2} / \mathrm{D}_{2}$. Or la clozapine offre un tel profil pharmacologique (rapport d'affinités 5- $\mathrm{HT}_{2} / \mathrm{D}_{2}$ élevé). De plus, les observations expérimentales préliminaires sur la clozapine présentées ici sont en accord avec les observations faites en électrophysiologie animale (voir plus haut) où l'on a démontré que la 5-HT et la dopamine participent au filtrage des messages afférents vers le CPF respectivement par les récepteurs $D_{2}$ et les récepteurs $5-\mathrm{HT}_{2}[13,14]$. Il reste à rechercher une amélioration de l'attention sélective chez des patients schizophrènes chez lesquels la neurotransmission noradrénergique aura été diminuée, puisque que la noradrénaline augmenterait la valeur de tout signal, pertinent ou non, en inhibant l'activité neuronale spontanée de base du CPF (voir plus haut). Les observations cliniques existantes sont en faveur de cette possibilité [30, 31]

\section{TIRÉS À PART}

R. Gedbout 


\section{Summary}

Prefrontal cortex, monoamines and neuropsychological studies in schizophrenia

The prefrontal cortex (PFC) plays a critical role in the temporal organization of motivated behavioral sequences, in cognitive processes such as representational memory, and in the regulation of emotional states. The PFC is also an area where the three main ascending monoaminergic systems converge, namely dopamine, serotonine (5-HT), and noradrenaline. It has been shown that these monoaminergic neurotransmitters, each in its own way, determine whether PFC neurons are receptive or not to incoming messages. In the normal waking state, this permits the brain to segregate essential information from accessory events. In thought disorders such as schizophrenia, this mechanism is deficient, but normal information processing can be reinstated by means of monoaminergic-active neuroleptics, as shown by preliminary results presented here.

\section{Remerciements}

Les auteurs tiennent à remercier le Fonds de la recherche en santé du Québec qui attribue à E.S. une bourse de chercheur-boursier clinicien du Centre de recherche Fernand-Seguin, hôpital Louis-H. Lafontaine et à R.G. une bourse de chercheur-boursier Junior II. Ils remercient également Isabelle Lussier, boursière post-doctorale du Conseil de la recherche médicale du Canada, pour l'aide à la mise au point de l'épreuve attentionnelle chez les patients schizophrènes. Les résultats de cette épreuve présentés ici ont été obtenus grâce à une subvention de la Fondation canadienne pour la recherche en psychiatrie. 